\title{
Design and Evaluation of a High Current Gain Resonant Inverter for Subsea Electrical Heating
}

This paper was downloaded from TechRxiv (https://www.techrxiv.org).

\section{LICENSE}

CC BY 4.0

SUBMISSION DATE / POSTED DATE

$15-10-2021 / 19-10-2021$

\section{CITATION}

Ray, Anindya; Kaushik, Rajashekara (2021): Design and Evaluation of a High Current Gain Resonant Inverter for Subsea Electrical Heating. TechRxiv. Preprint. https://doi.org/10.36227/techrxiv.16821163.v1

DOI

10.36227/techrxiv.16821163.v1 


\title{
Design and Evaluation of a High Current Gain Resonant Inverter for Subsea Electrical Heating
}

\author{
Anindya Ray, Member, IEEE, Kaushik Rajashekara, Fellow, IEEE,
}

\begin{abstract}
Direct electrical heating (DEH) is a well-established method for preventing hydrate formation inside subsea oil transfer pipelines. However, poor efficiency and high reactive power requirement of the existing line-frequency DEH systems have necessitated high-frequency power processing along with reactive power compensation. To meet these design objectives, a dc-ac converter using an LCCL resonant tank is presented in this paper to function as a high-frequency alternating current source. The LCCL resonant tank is tuned at the frequency of the peak tank current gain to maximize the heat generation and reduce the VA rating of the tank. The peak current gain operation also ensures zero voltage switching (ZVS) of the bridge inverter devices. Detailed frequency domain analysis and design guidelines are presented for the proposed LCCL resonant inverter (RI). Experimental results on a $10 \mathrm{~A}$ laboratory prototype and hardware-in-the-loop results for a 350 A system illustrate the advantages of the LCCL RI in DEH application.
\end{abstract}

Index Terms-Direct electrical heating (DEH), LCCL, immittance converter, constant current, peak current gain, zero voltage switching (ZVS).

\section{INTRODUCTION}

$\mathbf{R}$ ELIABILITY of the oil and gas (O\&G) transportation from the subsea is mostly affected by hydrate formation inside the oil transfer pipelines at low temperatures. The most economical solution to prevent hydrate formation is by direct electrical heating (DEH), which uses the heating effect of electric current to keep the flow temperature above the critical value of $25^{\circ} \mathrm{C}$ [1]-[3]. A typical direct electric heating system, shown in Fig. 1, utilizes a line-frequency 3-phase source in conjunction with a transformer and a load balancing network to circulate a single-phase alternating current through the metallic wall of the subsea pipeline [1], [2]. The resulting $I^{2} R$ loss maintains the pipeline flow at the required operating temperature. The pipeline is electrically represented by a single-phase $R L$ load.

$\mathrm{DEH}$ is a relatively new technology in the subsea O\&G industry, with the first DEH system implemented at the Asgard

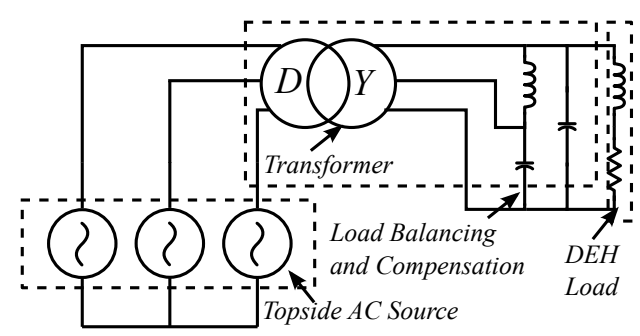

Fig. 1. Electrical network of a line-frequency DEH System [1], [2] field in Norway in the year 2000 [4]. Over the next two decades, two types of DEH technology have been explored, namely, DEH for wet insulated pipeline (DEH-WIP) and DEH for pipe-in-pipe (DEH-PIP). DEH-WIP is the most fieldproven DEH technology in subsea production, where the heating network is electrically connected to the surrounding seawater through several electrodes along the length of the pipe. In DEH-PIP, the metal pipe is insulated by another co-axial pipe and segmented into several closed electrical circuits through steel bulkheads. Due to the elimination of current leakage, DEH-PIP exhibits higher efficiency compared to DEH-WIP [4]. Nonetheless, both these line-frequency DEH systems manifest several disadvantages from the perspective of power supply to the heating system.

Low power factor is the main drawback of existing DEH systems. The metallic pipe, made of carbon steel, has a substantial $X / R$ ratio that causes a large reactive power demand for pipelines having a length of $25-50 \mathrm{~km}$ [5]. This increases the VA rating of the ac source and necessitates the installation of reactive compensation units, elevating the operating cost. The other disadvantage of line-frequency operation is suboptimal heat generation as the conduction power loss is the sole heat contributor. A higher operating frequency improves the DEH system efficiency due to the increased hysteresis loss in the carbon steel. The contribution of the hysteresis loss is estimated to be around $30 \%$ of the generated heat at the operating frequency of $200 \mathrm{~Hz}$ [6], [7].

State-of-the-art subsea electrification focuses on the direct current power architecture with underwater dc distribution [8], [9]. This presents a strong opportunity for an improved high-frequency DEH solution using load resonant converters. Resonant converters have been implemented in various highfrequency applications such as wireless power transfer [10][12], LED drivers [13], underwater power distribution [14], [15], etc. due to their soft-switching capability and high efficiency. Higher-order resonant topologies also exhibit a desirable property termed immittance. An immittance converter converts a voltage source into a current source and thus is widely used in load-independent constant current applications, such as charging power supply [16]-[19] and induction heating [20]-[23]. As DEH is electrically equivalent to induction heating, resonant inverters (RIs) emerge as the most feasible solution in high-frequency subsea pipeline heating.

The rest of this paper is organized as follows. Section II examines the choice of the RIs for DEH systems. The characteristics of the LCCL tank prove to be more suitable for the large inductance of the DEH load. The steady-state modeling and analysis of the full-bridge LCCL RI are presented in 


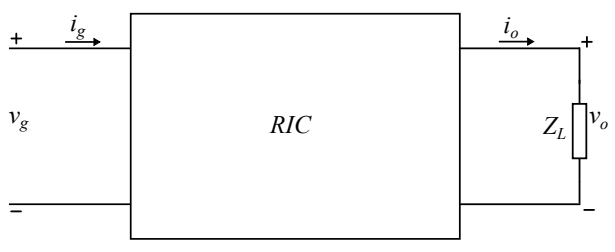

(a)

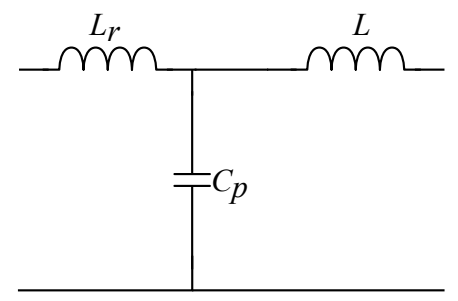

(b)

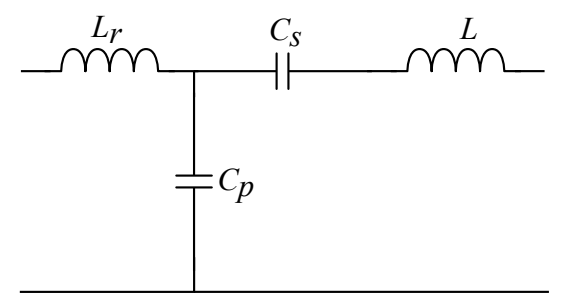

(c)

Fig. 2. (a) Two-port RIC, (b) LCL-T, (c) LCCL [16].

section III. The converter design procedure for the operation at the frequency of peak current gain is discussed in section IV. Experimental results on a 10 A prototype and hardware-inthe-loop (HIL) results for a 350 A DEH system are presented in section $\mathrm{V}$ to demonstrate the performance of the proposed RI. The principal outcomes in this paper are summarized in section VI.

\section{RESONANT INVERTER SELECTION FOR DEH}

Resonant inverters are extensively utilized in constant current applications like induction heating due to the immittance property. An immittance converter is a generic two-port network having the input impedance proportional to the load admittance [16]. The two-port resonant immittance converter (RIC), shown in Fig. 2(a), is defined by the following equation

$$
\left[\begin{array}{l}
v_{g} \\
i_{g}
\end{array}\right]=\left[\begin{array}{cc}
0 & \mp j Z_{o c} \\
\pm j \frac{1}{Z_{o c}} & 0
\end{array}\right] \cdot\left[\begin{array}{c}
v_{o} \\
-i_{o}
\end{array}\right]
$$

where $Z_{o c}$ is the characteristic impedance of the RIC. It is apparent from (1), that the output current $\left(i_{o}\right)$ of the RIC is proportional to the input voltage $\left(v_{g}\right)$, i.e., it can convert a constant voltage source to a constant-current sink. The immittance property is observed in resonant topologies with three or more tank elements.

Amongst multi-element resonant converters, LCL-T is the simplest and the most frequently used tank topology [17]-[19], [21]-[23]. The immittance condition for the LCL-T topology, shown in Fig. 2(b), is given by,

$$
\omega L_{r}=\omega L=\frac{1}{\omega C_{p}}
$$

which can be further simplified as

$$
\omega=\omega_{o}, \quad \beta=1
$$

where $\beta$ represents the tank inductor ratio and $\omega_{0}$ is the resonant frequency of the tank. These parameters are expressed as,

$$
\beta=\frac{L}{L_{r}}, \quad \omega_{o}=\frac{1}{\sqrt{L_{r} C_{p}}}
$$

The LCL-T RI at the immittance condition given by (3) operates with the maximum current gain and the minimum tank rms current at unity power factor. However, these advantages are difficult to realize for a DEH system due to the large inductance of the DEH pipeline load. For $\beta>1$, the LCL-T topology exhibits a low power factor with significantly higher tank rms current for the operation at the resonant frequency.
The above issues of the LCL-T topology for highly reactive loads are mitigated by utilizing the LCCL tank topology, shown in Fig. 2(c). The immittance condition for the LCCL network is expressed as,

$$
\omega=\omega_{o}, \quad \beta=1+\frac{1}{\alpha}, \quad \alpha=\frac{C_{s}}{C_{p}}
$$

where $\alpha$ denotes the tank capacitor ratio. The LCCL RI exhibits similar advantages as the LCL-T RI when the immittance conditions in (5) are satisfied. However, even for nonimmittance, the LCCL RI can be designed to operate with high current gain and reduced tank rms current, as presented in [1]. Therefore, a full-bridge LCCL RI is analyzed in this paper for the implementation in high-frequency DEH systems. The presented topology is designed at the frequency of peak tank current gain, which minimizes the tank VA rating and improves the system efficiency.

\section{Steady State Analysis of LCCL RI}

Fig. 3(a) shows the LCCL resonant inverter which converts a constant dc input voltage to an alternating current of constant amplitude. The full-bridge network, comprising of the MOSFETs $Q_{1}-Q_{4}$, is modulated with a generic phase shift angle of $\varphi_{p}$. The square wave duty cycle modulation is a limiting case with $\varphi_{p}=180^{\circ}$. The quasi-square wave (QSW) voltage at the output of the inverter drives the LCCL resonant tank formed by inductors $L_{r}$ and $L$, and capacitors $C_{p}$ and $C_{s}$. The distributed impedance of the metal pipeline is modeled as an $R L$ load. An $N: 1$ isolation transformer may be used following the tank network for further amplifying the current gain.

Applying the first harmonics approximation (FHA) [24], the fundamental frequency equivalent circuit of the LCCL RI is constructed in Fig. 3(b). This equivalent circuit is further simplified as the steady-state ac network in Fig. 3(c) [14], [25]. The dc input current is expressed as,

$$
I_{g}=\frac{2}{\pi} I_{s_{1}} \sin \left(\frac{\varphi_{p}}{2}\right) \cos \left(\varphi_{i}\right),
$$

where $\varphi_{i}$ represents the tank power factor angle, i.e. $\varphi_{i}=\angle Z_{i}$. $Z_{i}$ represents the input impedance of the loaded resonant tank. $I_{s_{1}}$ is the amplitude of the fundamental component of the tank input current. $\omega_{s}$ denotes the angular switching frequency of operation. 


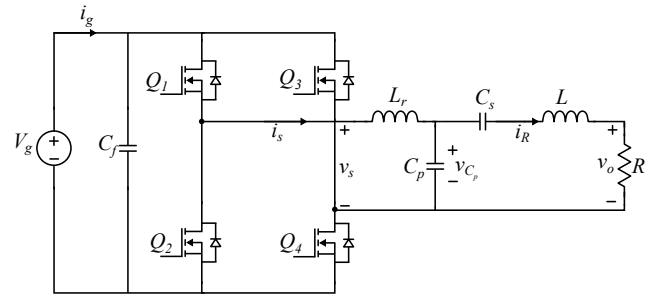

(a)

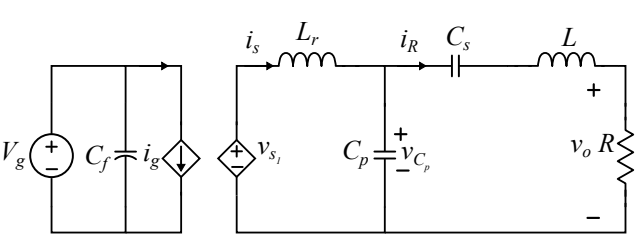

(b)

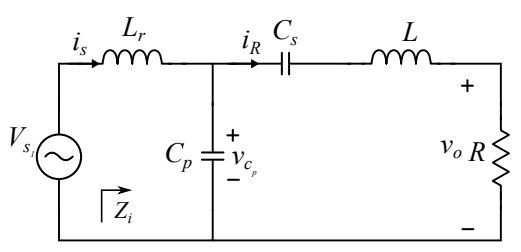

(c)

Fig. 3. LCCL resonant inverter. (a) Schematic, (b) FHA equivalent circuit, (c) Simplified ac model.

\section{A. Operating Point Selection}

The tank current gain is evaluated from the equivalent circuit in Fig. 3(b) as,

$$
H_{i}(s)=\frac{I_{R}(s)}{I_{s}(s)}=\frac{1}{\beta \frac{s^{2}}{\omega_{o}^{2}}+Q \frac{s}{\omega_{o}}+\left(1+\frac{1}{\alpha}\right)}
$$

where $Q$ represents the quality factor of the loaded resonant tank and is expressed as,

$$
Q=\frac{R}{Z_{o}}, \quad Z_{o}=\sqrt{\frac{L_{r}}{C_{p}}}
$$

$Z_{o}$ represents the characteristic impedance of the resonant tank. The magnitude of the tank current gain at the switching frequency $f_{s}$ is evaluated from (7) as,

$$
\left|H_{i}\left(j \omega_{s}\right)\right|=\frac{1}{\sqrt{\left[\left(1+\frac{1}{\alpha}\right)-\beta F^{2}\right]^{2}+Q^{2} F^{2}}}
$$

where $F=\frac{f_{s}}{f_{o}}=\frac{\omega_{s}}{\omega_{o}}$ is the normalized switching frequency w.r.t the resonant frequency $f_{o}$. It is observed from (9) that the maximum tank current gain is realized for the frequency

$$
F=F_{m}=\sqrt{\frac{\alpha+1}{\alpha \beta}}
$$

Furthermore, the maximum current gain is obtained at $F=1$, if the immittance condition in (5) is fulfilled. The tank current gain $\left(H_{i}\right)$ is plotted against the normalized switching frequency $(F)$ for various loads $(Q)$ with two different capacitance ratios. Fig. 4(a) shows the variation of $H_{i}$ for $\alpha=1$ and $\beta=2$, which satisfies (5). Hence, $H_{i}$ attains its maximum value at $F=1$ for the corresponding $Q$ while realizing the minimum tank rms current.

Despite being the optimum operating point of the LCCL RI, immittance is difficult to realize for the highly inductive DEH load. In such a scenario, the condition given by equation (10) proves to be the most feasible quiescent point. Fig. 5(a) shows the plot of $H_{i}$ versus $F$ variation with $\alpha=0.5$ and $\beta=2$. The maximum current gain for a specific $Q$ value is obtained at $F_{m}=1.22$ in accordance with (10). Thus, for a given load current, the rms tank current $\left(I_{s_{r}}\right)$ is minimized.

The operation of the LCCL RI at the frequency of the maximum tank current gain $\left(F_{m}\right)$ is further justified from the plot of the converter input impedance $Z_{i}$, which is expressed in the Laplace domain as

$$
Z_{i}(s)=Z_{o} \frac{\alpha \beta \frac{s^{4}}{\omega_{o}^{4}}+\alpha Q \frac{s^{3}}{\omega_{o}^{3}}+(1+\alpha+\alpha \beta) \frac{s^{2}}{\omega_{o}^{2}}+\alpha Q \frac{s}{\omega_{o}}+1}{\alpha \beta \frac{s^{3}}{\omega_{o}^{3}}+\alpha Q \frac{s^{2}}{\omega_{o}^{2}}+(1+\alpha) \frac{s}{\omega_{o}}}
$$

The magnitude of $Z_{i}$ at the switching frequency $f_{s}$ is found to be

$$
\left|Z_{i}\left(j \omega_{s}\right)\right|=Z_{o} \frac{Z_{R}+j Z_{I}}{\left(\alpha Q F^{2}\right)^{2}+F^{2}\left(1+\alpha-\alpha \beta F^{2}\right)^{2}}
$$

where, $Z_{R}$ and $Z_{I}$ are the real and imaginary components of the input impedance, respectively.

$$
Z_{R}=Q F^{2}\left[1-F^{2}(1-\alpha)(1+\alpha+\alpha \beta)+F^{4} \alpha \beta(1-\alpha)\right]
$$

and,

$$
\begin{array}{r}
Z_{I}=-F(1+\alpha)+F^{3}\left[\alpha\left(\beta-Q^{2}\right)+(1+\alpha)(1+\alpha+\alpha \beta)\right] \\
+F^{5} \alpha\left[Q^{2}-\beta(2+2 \alpha+\alpha \beta)\right]+\alpha^{2} \beta^{2} F^{7}
\end{array}
$$

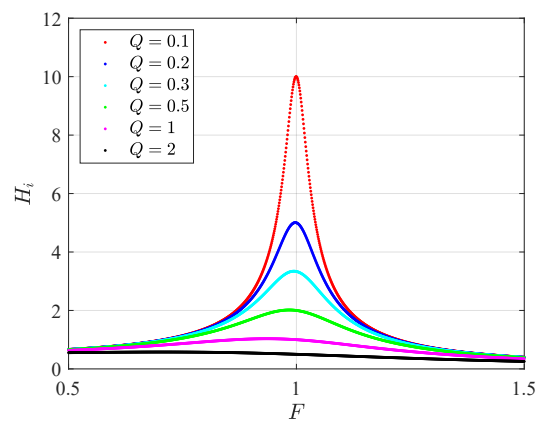

(a)

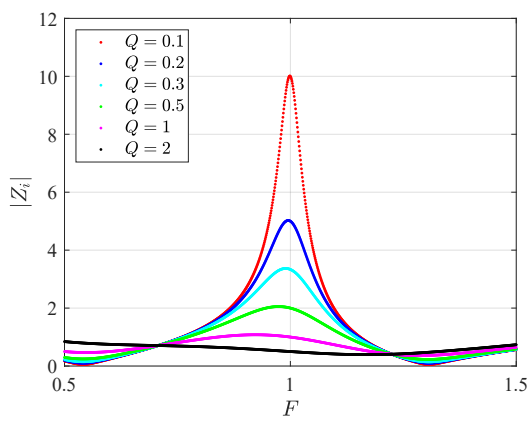

(b)

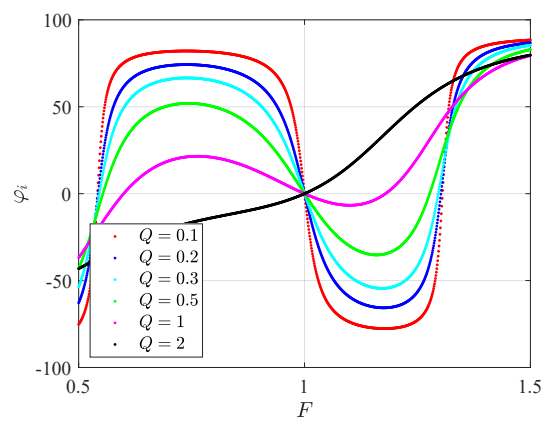

(c)

Fig. 4. LCCL RI characteristics. (a) Tank Current Gain $\left(H_{i}\right)$, (b) Tank input impedance $\left(Z_{i}\right)$, (c) Power factor angle $\left(\varphi_{i}^{\circ}\right)$ versus normalized switching frequency $(F)$ for different loads $(Q)$, with $\alpha=1, \beta=2$ 


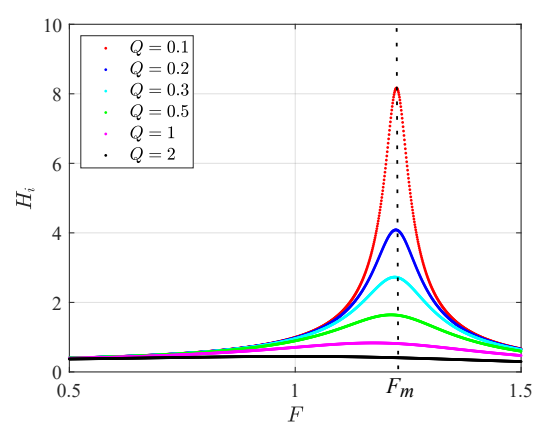

(a)

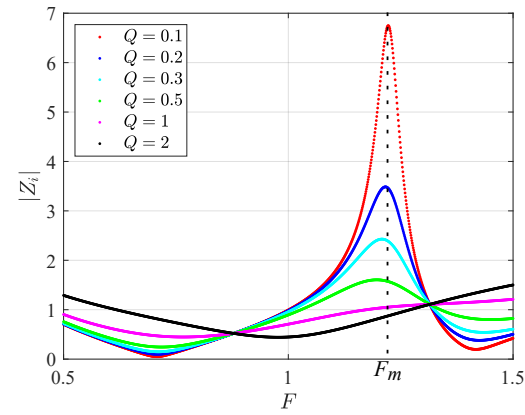

(b)

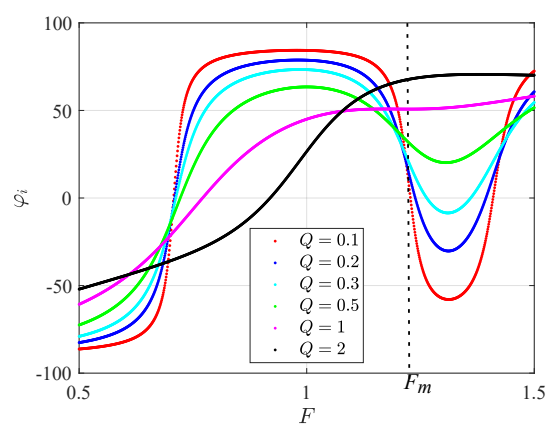

(c)

Fig. 5. LCCL RI characteristics. (a) Tank Current Gain $\left(H_{i}\right)$, (b) Tank input impedance $\left(Z_{i}\right)$, (c) Power factor angle $\left(\varphi_{i}^{\circ}\right)$ versus normalized switching frequency $(F)$ for different loads $(Q)$, with $\alpha=0.5, \beta=2$

Fig. 4(b) shows the variation of the normalized input impedance $Z_{i, n}=\frac{\left|Z_{i}\right|}{Z_{o}}$ with $F$ for different loads at the immittance condition, i.e. $\alpha=1$. It is observed that $Z_{i, n}$ attains its maximum at $F=1$ for low $Q$ values, which implies that the corresponding tank input current is minimized. As $Q$ increases, $Z_{i, n}$ shows minimal change with $F$ variation. Fig. 5(b) presents the input impedance profile for the nonimmittance condition with $\alpha=0.5 . Z_{i, n}$ is at or near its maximum value at $F_{m}$ for low $Q$ values. Hence, at the switching frequency $F_{m}$, the LCCL RI still operates with the minimum tank rms tank current, provided the load $Q$ variation is limited.

\section{B. Soft Switching}

The soft switching condition of the MOSFETs $Q_{1}-Q_{4}$ is evaluated using the mathematical expression of the tank power factor angle $\varphi_{i}$, which is given by,

$$
\varphi_{i}=\tan ^{-1}\left(\frac{Z_{I}}{Z_{R}}\right)
$$

Fig. 4(c) shows the $\varphi_{i}-F$ characteristics for $\alpha=1$. At immittance, $\varphi_{i}=0^{\circ}$ for all $Q$ at $F=1$. This represents the operation of the inverter at unity power factor (UPF), which results in the lowest tank conduction loss. However, UPF operation does not guarantee ZVS of all the switches.

On the other hand, the operation at $F_{m}$ results in a positive $\varphi_{i}$, implying lagging power factor operation of the inverter. For $\varphi_{i}>0$, the antiparallel diodes conduct before the corresponding MOSFETs, resulting in their ZVS turn-on. The phase angle is small for low $Q$ values at $F_{m}$, as seen from Fig. 5(c), indicating high power factor operation of the inverter. However, an increase in $Q$ reduces the tank power factor, causing the inverter to draw more reactive current. Therefore, the optimum performance at $F=F_{m}$ is realized for a limited variation in the load resistance, which mimics the DEH load profile.

\section{Design of LCCL Ri for Peak Current Gain}

\section{A. Tank Design}

With the selected operating point of $F=F_{m}$, the series inductor $L_{r}$ and the capacitors $C_{p}$ and $C_{s}$ are designed to

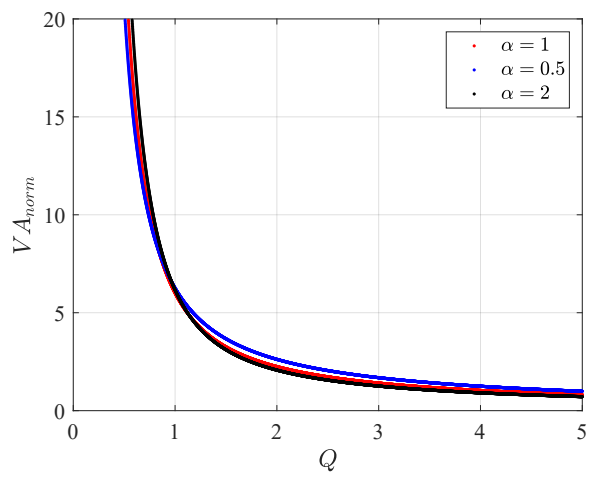

Fig. 6. Normalized VA rating of LCCL RI as a function of $Q$.

minimize the cumulative VA rating of the resonant tank. This, in turn, reduces the converter volume which is desirable for subsea operation within pressure chambers. The impedances of the individual tank elements in the equivalent circuit of Fig. 3(b) are evaluated at $F_{m}$ as,

$$
\begin{gathered}
X_{L_{r}}=2 \pi f_{s} L_{r}=F_{m} Z_{o}, X_{C_{p}}=\frac{1}{2 \pi f_{s} C_{p}}=\frac{Z_{o}}{F_{m}}, \\
X_{C_{s}}=\frac{1}{2 \pi f_{s} C_{s}}=\frac{Z_{o}}{\alpha F_{m}}, X_{L}=2 \pi f_{s} L=\beta F_{m} Z_{o}
\end{gathered}
$$

The rms value of the fundamental component of the tank input current is expressed from equation (6) as

$$
I_{s_{r}}=\frac{\pi}{2 \sqrt{2}} \frac{I_{g}}{\sin \left(\frac{\varphi_{p}}{2}\right) \cos \left(\varphi_{i}\right)}
$$

The rms values of the tank input current $\left(i_{s}\right)$ and the load current $\left(i_{R}\right)$ are also related as,

$$
I_{s_{r}}=\frac{I_{R_{r}}}{H_{i}}
$$

The rms current through the parallel capacitor $C_{p}$ is expressed as,

$$
I_{C_{p r}}=\sqrt{I_{s_{r}}^{2}+I_{R_{r}}^{2}}=I_{R_{r}} \sqrt{1+\frac{1}{H_{i}^{2}}}
$$




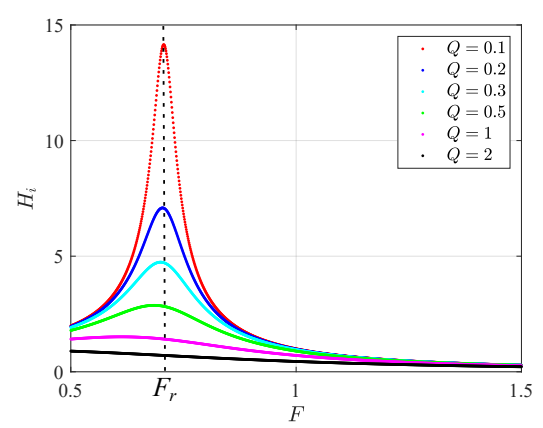

(a)

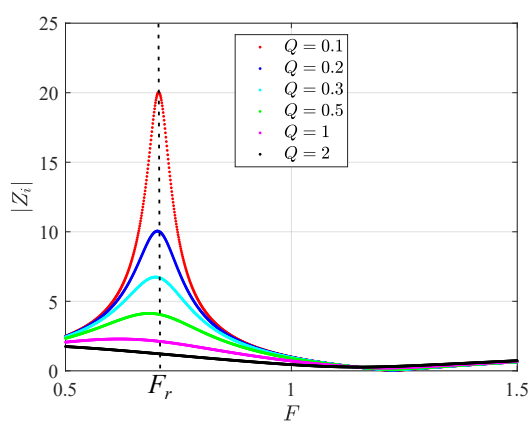

(b)

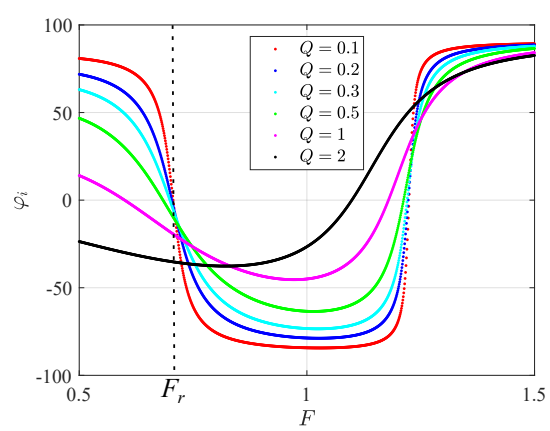

(c)

Fig. 7. LCL-T RI characteristics. (a) Current gain, (b) Normalized input impedance, (c) phase angle versus normalized switching frequency ( $F$ ) for different loads $(Q)$, with $\beta=2$

Using (16)-(19), the VA rating of the resonant tank components are computed as

$$
\begin{gathered}
V A_{L_{r}}=I_{s_{r}}^{2} X_{L_{r}}=\frac{I_{R_{r}}^{2}}{H_{i}^{2}} F_{m} Z_{o} \\
V A_{C_{p}}=I_{C_{p r}}^{2} X_{C_{p}}=I_{R_{r}}^{2}\left(1+\frac{1}{H_{i}^{2}}\right) \frac{Z_{o}}{F_{m}} \\
V A_{C_{s}}=I_{R_{r}}^{2} X_{C_{s}}=I_{R_{r}}^{2} \frac{Z_{o}}{\alpha F_{m}} \\
V A_{L}=I_{R_{r}}^{2} X_{L}=I_{R_{r}}^{2} \beta F_{m} Z_{o}
\end{gathered}
$$

The tank current gain at $F_{m}$ is evaluated to be

$$
\left.H_{i}\right|_{F=F_{m}}=\frac{1}{Q F_{m}}
$$

The cumulative VA of the tank is the sum of VA ratings of the tank components, i.e.

$$
V A_{\text {tank }}=\frac{P_{o}}{Q}\left[\frac{1}{Q^{2} F_{m}}\left(1+\frac{1}{F_{m}^{2}}\right)+\frac{1}{F_{m}}\left(1+\frac{1}{\alpha}\right)+F_{m} \beta\right]
$$

where $P_{o}$ represents the effective heating power without considering the hysteresis loss, and is expressed as,

$$
P_{o}=I_{R_{r}}^{2} R=I_{R_{r}}^{2} Q Z_{o}
$$

Hence, the normalized VA rating of the tank is expressed as

$$
V A_{\text {norm }}=\frac{1}{Q}\left[\frac{1}{Q^{2} F_{m}}\left(1+\frac{1}{F_{m}^{2}}\right)+\frac{1}{F_{m}}\left(1+\frac{1}{\alpha}\right)+F_{m} \beta\right]
$$

The normalized tank VA is plotted against $Q$ values with $\alpha=0.5,1,2$ and $\beta=2$ in Fig. 6. The deviation in tank VA is not significant between immittance $(\alpha=1)$ and nonimmittance $(\alpha=0.5,2)$. Also, $V A_{\text {norm }}$ is observed to reduce significantly with larger $Q$ values. However, Fig. 5(c) shows that the tank power factor reduces for large $Q$ values at non-immittance conditions. As the metal pipeline has a large $X / R$ ratio, the nominal $Q$ value is small in a DEH system. Consequently, a choice of $Q=1-2$ yields the optimum performance at the switching frequency $\left(F_{m}\right)$.

The rms voltages across the tank capacitors can be computed as

$$
V_{C_{p r}}=I_{C_{p r}} X_{C_{p}}=\frac{V_{o_{r}}}{Q F_{m}} \sqrt{1+\frac{1}{\left(Q F_{m}\right)^{2}}}
$$

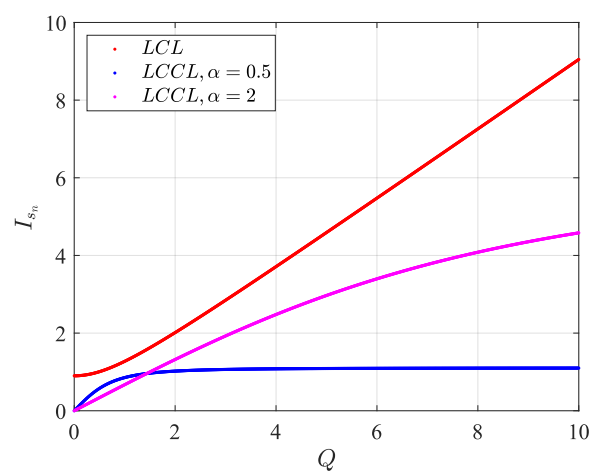

Fig. 8. Normalized rms tank currents of LCL-T and LCCL RI, $\beta=2$

and

$$
V_{C_{s r}}=I_{R_{r}} X_{C_{s}}=\frac{V_{o_{r}}}{Q F_{m}}
$$

where $V_{o_{r}}=I_{R_{r}} R$ denotes the rms load voltage.

The first step of the tank design involves determining the load parameters $L$ and $R$. Thereafter, $\beta$ and $Q$ are chosen to determine $L_{r}$ and $C_{p}$ from (4). Finally, $\alpha$ is selected to compute $C_{s}$ and the switching frequency $F_{m}$. Equations (17)(19) and (28)-(29) provide the component ratings.

\section{B. Comparison with LCL-T RI}

To further establish the feasibility of the LCCL RI for DEH systems, a brief evaluation of the LCL-T RI for the same application is presented here. $\beta$ is greater than unity owing to the large value of $L$. The tank current gain of the LCL-T is given by

$$
\left|H_{i}\right|=\frac{1}{\sqrt{\left(1-\beta F^{2}\right)^{2}+Q^{2} F^{2}}}
$$

Consequently, the maximum $\left|H_{i}\right|$ is realized for $F=F_{r}=$ $\frac{1}{\sqrt{\beta}}$. This results in an operating frequency lower than the resonant frequency $f_{o}$, as shown in Fig. 7(a). On the other hand, the maximum $\left|H_{i}\right|$ of the LCCL RI occurs at $f_{s}>f_{o}$ for $\alpha<1$ and the same $\beta$. 
TABLE I

PARAMETERS OF THE LCCL RI PROTOTYPE

\begin{tabular}{ccc}
\hline \hline Parameters & Symbol & Value \\
\hline Rated Load Current & $I_{R_{r}}$ & $10 \mathrm{~A}$ \\
Tank Inductor & $L_{r}$ & $310.21 \mu \mathrm{H}$ \\
Parallel Capacitor & $C_{p}$ & $9.77 \mu \mathrm{F}$ \\
Series Capacitor & $C_{s}$ & $4.9 \mu \mathrm{F}$ \\
Load Inductor & $L$ & $622.08 \mu \mathrm{H}$ \\
Load Resistor & $R$ & $2.37 \Omega$ \\
Resonant frequency & $f_{o}$ & $2891 \mathrm{~Hz}$ \\
Peak Current Gain frequency & $f_{m}$ & $3531 \mathrm{~Hz}$ \\
\hline \hline
\end{tabular}

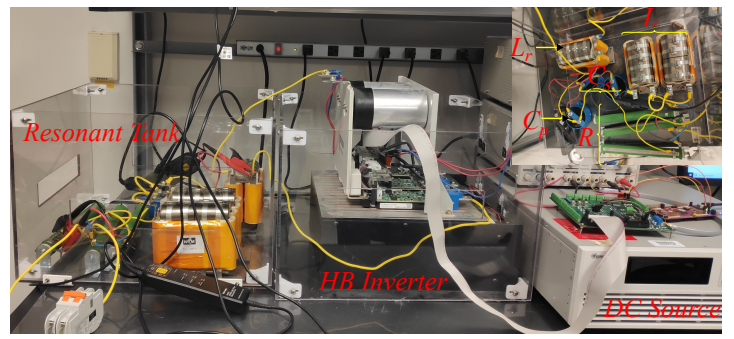

Fig. 9. Experimental prototype of the LCCL RI

Also, the normalized input impedance magnitude and phase of LCL-T RI is expressed as

$$
\left|Z_{i}\right|=\frac{Z_{r e}+j Z_{i m}}{\left(1-\beta F^{2}\right)^{2}+Q^{2} F^{2}}, \quad \varphi_{i}=\tan ^{-1}\left(\frac{Z_{i m}}{Z_{r e}}\right)
$$

where $Z_{r e}$ and $Z_{i m}$ are the real and imaginary parts of $Z_{i}$, respectively, and are given by

$$
\begin{aligned}
& Z_{r e}=Q\left(1-F^{2}\right)\left(1-\beta F^{2}\right)+Q F^{2}\left[(1+\beta)-\beta F^{2}\right] \\
& Z_{i m}=F\left(1-\beta F^{2}\right)\left[(1+\beta)-\beta F^{2}\right]-Q^{2} F\left(1-F^{2}\right)
\end{aligned}
$$

The $Z_{i}-F$ characteristics of LCL-T RI is plotted in Fig. 7(b)(c) for $\beta=2$. The magnitude plot in Fig. 7(b) reveals that $\left|Z_{i}\right|$ is minimum at $F=1$, implying an increase in the rms tank input current if the inverter is switched at $f_{o}$. Also, the power factor angle is negative at $F=1$ as per Fig. 7(c), which means the MOSFETs $Q_{1}-Q_{4}$ undergo ZCS instead of ZVS.

Fig. 8 compares the normalized tank input current of the LCL-T and the LCCL RI, given by

$$
I_{s_{n}}=\frac{I_{s_{r}}}{\frac{V_{g}}{Z_{o}}}=\frac{2 \sqrt{2}}{\pi\left|Z_{i}\right|}
$$

It is observed that the LCL-T RI with $\beta=2$ realizes the maximum $I_{s_{n}}$ operating at $F=1$. An increasing $I_{s_{n}}$ profile is also observed in the case of the LCCL-RI for $\alpha=2$ and $F=1$. So, both LCL-T and LCCL RI would incur higher conduction loss when operating at the resonant frequency. However, with $\alpha=0.5$ and $F=F_{m}, I_{s_{n}}$ is minimized. Thus the peak current gain operation of the LCCL RI yields the minimum tank rms current, in line with equations (9) and (12).

\section{Performance Evaluation of LCCL RI}

\section{A. Experimental Results}

A laboratory prototype is constructed to evaluate the performance of the LCCL RI for various immittance and non-

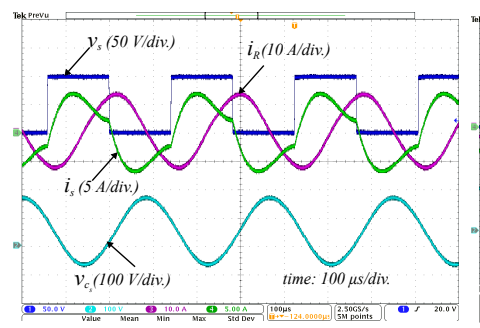

(a)

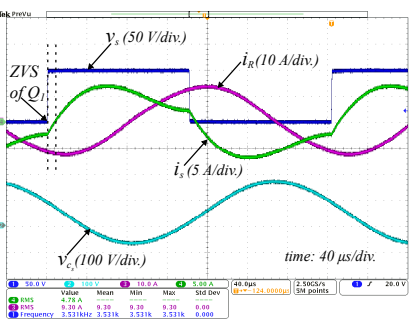

(b)
Fig. 10. Half-bridge LCCL RI at $f_{s}=f_{m}$, (a) Steady-state waveform at $V_{g}=100 \mathrm{~V}$, (b) ZVS of $Q_{1}$

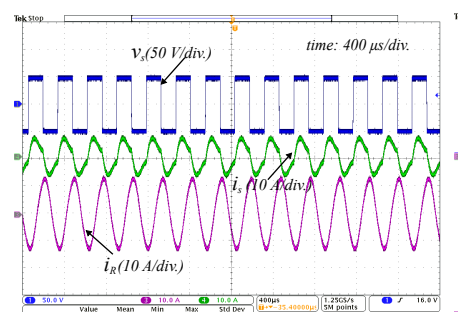

(a)

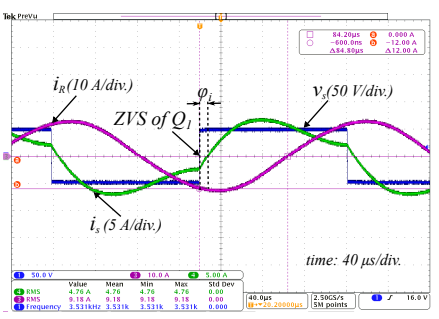

(b)
Fig. 11. Full-bridge LCCL RI at $f_{s}=f_{m}$, (a) Steady-state waveform at $V_{g}=50 \mathrm{~V}$, (b) ZVS of $Q_{1}$.

immittance operating points. The complete experimental system is shown in Fig. 9. The converter and load parameters are listed in Table I, following the design procedure in section IV. The DEH load is emulated by discrete resistors and inductors. Due to presence of the series tank capacitor $C_{s}$, the LCCL RI can be tested in both half-bridge and full-bridge configurations, without the need of an additional dc blocking capacitor. The LCL-T RI is also tested using the same setup, but only in full-bridge configuration. The bridge inverter is implemented by an AgileSwitch SiC power module. A Chroma $62150 \mathrm{H}-$ 1000 power supply is utilized as the dc source. The $50 \%$ duty cycle based modulation scheme is implemented by a TI TMS320F28379D DSP to maximize the inverter output. The gate-driving stage is realized in an ALTERA MAX-II CPLD based control board.

The tank voltage and current waveforms for the half-bridge LCCL RI, switching at the frequency of the peak tank current gain $\left(f_{m}\right)$, is shown in Fig. 10. The source voltage $V_{g}$ is 100 V. It is observed from Fig. 10(a) that the tank input current $i_{s}$ is negative at the rising edge of the inverter output voltage $v_{s}$. This implies soft-switching of the high-side half-bridge MOSFET $Q_{1}$. The ZVS turn-on of $Q_{1}$ is further illustrated over an amplified time scale in Fig. 10(b). The negative $i_{s}$ is conducted by the anti-parallel diode of $Q_{1}$ to enable ZVS of $Q_{1}$. Also, the lag angle $\varphi_{i}$ is around $10^{\circ}$, which implies a high power factor operation of the LCCL RI. The rms tank current $\left(I_{s_{r}}\right)$ is measured to be $4.83 \mathrm{~A}$ corresponding to a rms load current $\left(I_{R_{r}}\right)$ of $9.3 \mathrm{~A}$, which denotes the minimum rms current operation, as illustrated in Fig. 8.

Fig. 11 shows the same voltage and current waveforms for the full-bridge LCCL RI for a dc source voltage $\left(V_{g}\right)$ of 50 V. $V_{g}$ is limited by the power rating of the load resistor bank. 


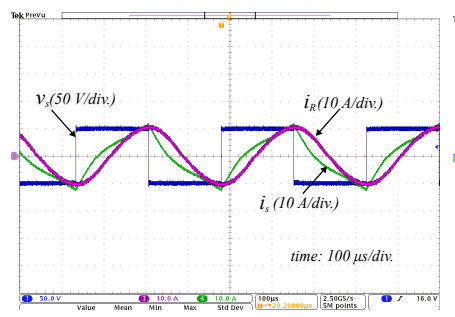

(a)

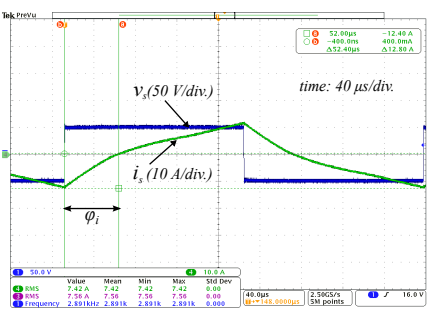

(b)
Fig. 12. Full-bridge LCCL RI at $f_{s}=f_{o}$, (a) Steady-state waveform at $V_{g}=50 \mathrm{~V}$, (b) Increase in power factor angle $\varphi_{i}$.

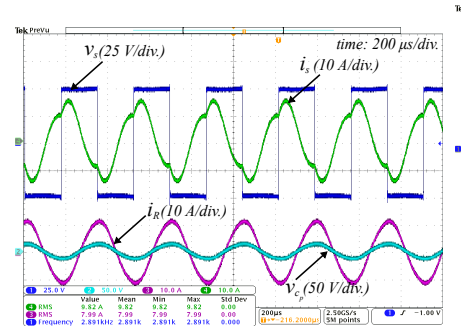

(a)

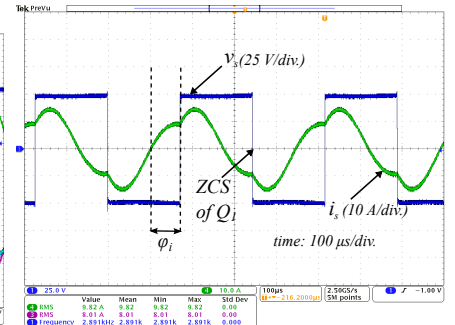

(b)
Fig. 13. Full-bridge LCL-T RI at $f_{s}=f_{o}$, (a) Steady-state waveform at $V_{g}=50 \mathrm{~V}$, (b) Tank quantities over amplified time-scale

Fig. 11(a) provides the identical phase relation between $v_{s}$, $i_{s}$ and $i_{R}$. The peak current gain operation ensures ZVS of the full-bridge MOSFETs. ZVS turn-on of $Q_{1}$ is depicted in detail in Fig. 11(b). The tank power factor and the $I_{s_{r}}$ and $I_{R_{r}}$ values are almost identical to the half-bridge configuration. The measured tank current gain is equal to $\frac{1}{F Q}$ as per (9). The only difference from the half-bridge operation is observed in the absence of dc offset in the inverter output voltage and the capacitor voltages.

The advantages of the peak tank current gain operation is further demonstrated by setting the switching frequency $f_{s}$ of the full-bridge LCCL RI to the resonant frequency $f_{o}$ with the dc source voltage unchanged. Fig. 12(a) shows the steadystate $v_{s}, i_{s}$, and $i_{R}$ waveforms at the resonant frequency. The phase angle of $i_{s}$ is observed to increase significantly, which increases the tank rms current $\left(I_{s_{r}}\right)$. Meanwhile, the rms load current $\left(I_{R_{r}}\right)$ is reduced. These observations are consistent with equations (12), (15), and (34) and the rms current plot in Fig. 8, as the tank input impedance $Z_{i}$ reduces to a small value at $f_{o}$. Fig. 12(b) presents the phase relationship of $v_{s}$ and $i_{s}$ over an amplified time scale. Power factor angle $\varphi_{i}$ is found to be almost $66^{\circ}$, which denotes a substantial increase in the reactive power drawn by the tank, causing an increase in the conduction loss. Thus, for a largely reactive heating load, the switching at $f_{m}$ provides the optimal efficient operation.

To compare the effectiveness of the proposed design, the full-bridge LCL-T RI with the parameters in Table I is tested at the resonant frequency $f_{o}$ with the dc source voltage $\left(V_{g}\right)$ of $50 \mathrm{~V}$. Fig. 13 depicts the corresponding tank waveforms. The tank input current $i_{s}$ leads the inverter output voltage $v_{s}$, as shown in Fig. 13(a). This event is consistent with (31) and Fig. 7(b)-(c). Also, the rms load current $\left(I_{R_{r}}\right)$ is lower than the

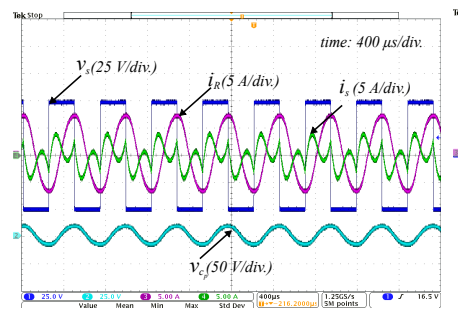

(a)

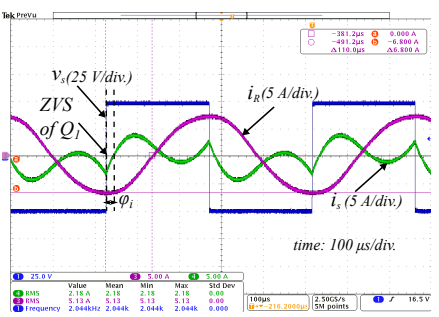

(b)
Fig. 14. Peak current gain operation of LCL-T RI, (a) Steady-state waveform at $V_{g}=50 \mathrm{~V}$, (b) Tank quantities over amplified time-scale.

TABLE II

PARAMETERS OF THE DEH SYSTEM

\begin{tabular}{ccc}
\hline \hline Parameters & Symbol & Value \\
\hline Rated Load Current & $I_{R_{r}}$ & $350 \mathrm{~A}$ \\
Tank Inductor & $L_{r}$ & $2.5 \mathrm{mH}$ \\
Parallel Capacitor & $C_{p}$ & $50 \mu \mathrm{F}$ \\
Series Capacitor & $C_{s}$ & $13.6 \mu \mathrm{F}$ \\
Load Inductor & $L$ & $9.2 \mathrm{mH}$ \\
Load Resistor & $R$ & $2 \Omega$ \\
Resonant frequency & $f_{o}$ & $450 \mathrm{~Hz}$ \\
Peak Current Gain frequency & $f_{m}$ & $507 \mathrm{~Hz}$ \\
\hline \hline
\end{tabular}

tank rms current $\left(I_{s_{r}}\right)$. The current gain is less than that of the LCCL RI operating at $f_{o}$. The leading power factor operation causes Zero current turn-off of the MOSFETs. The ZCS of MOSFET $Q_{1}$ is illustrated in Fig. 13(b) over an amplified time scale. The lead angle $\varphi_{i}$ is computed to be around $72^{\circ}$, which implies a large reactive power demand of the RI and a low system efficiency.

The LCL-T RI is also switched at the frequency of its peak current gain $\left(f_{r}\right)$, to provide a consistent comparison with the presented design. For the parameters given in Table I, $f_{r}$ is calculated to be $2044 \mathrm{~Hz}$ as per (30). Fig. 14(a) shows $v_{s}, i_{s}$ and $i_{R}$ along with the voltage across the shunt capacitor $C_{p}$. The lagging phase angle between $v_{s}$ and $i_{s}$ is small. Hence the LCL-T resonant tank operates with a high power factor, while the MOSFETs undergo ZVS turn-on. This event is highlighted over a magnified time scale in Fig. 14(b). The power factor angle $\left(\varphi_{i}\right)$ is around $15^{\circ}$. Although the current gain is high, $I_{R_{r}}$ is almost half of that of the LCCL RI in Fig. 11(b). Hence, significantly less heating output is realized by the peak current gain operation of the LCL-T RI compared to that of the LCCL RI for the same source voltage.

\section{B. Hardware-in-the-Loop Results}

A hardware-in-the-loop (HIL) simulation model of the proposed LCCL RI was implemented in Typhoon-HIL platform to verify its efficacy in high power DEH systems. The system parameters are tabulated in Table II. Multiple such RIs may be implemented in a distributed dc power architecture to realize the rated alternating current depending on the pipeline length [1]. The dc distribution bus voltage is maintained at $2.5 \mathrm{kV}$. Fig. 15(a) shows the steady-state inverter waveforms for the peak current gain operation. Similar to Fig. 11(a), the tank current $i_{s}$ lags the inverter output voltage by a small angle, indicating high tank power factor. ZVS turn-on of the 


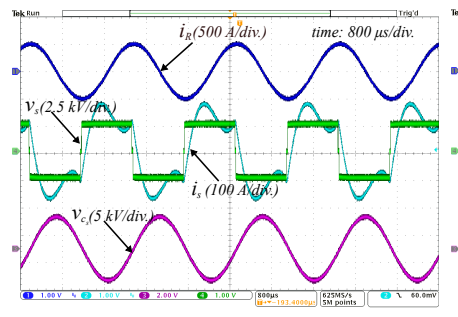

(a)

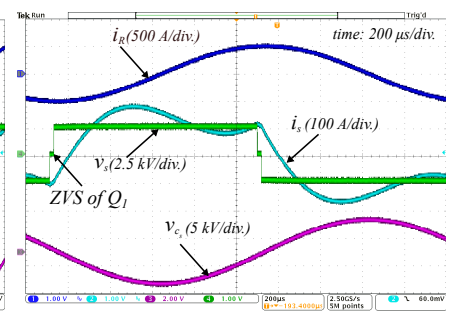

(b)
Fig. 15. LCCL RI for $I_{R_{r}}=350 \mathrm{~A}$, (a) Steady-state waveform at $V_{g}=2500 \mathrm{~V}$, (b) ZVS of $Q_{1}$.

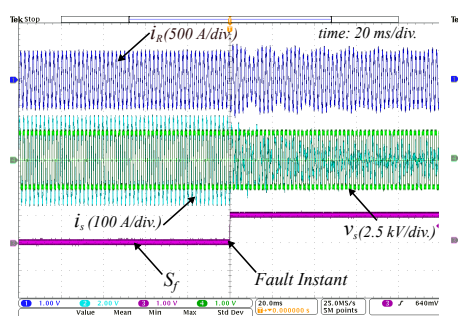

(a)

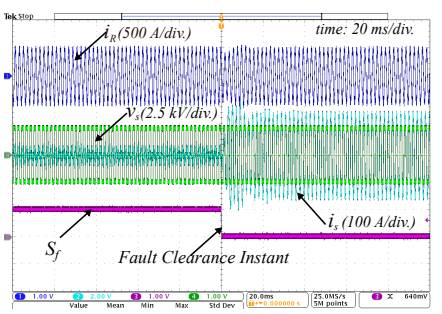

(b)
Fig. 16. Fault tolerant operation of LCCL RI (a) Fault initiated, (b) Fault cleared.

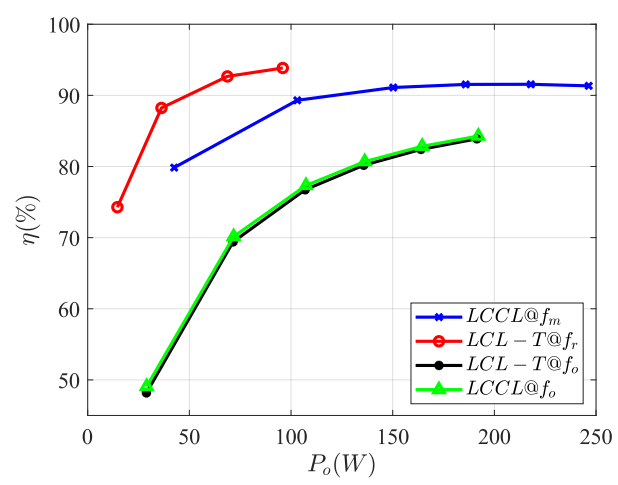

Fig. 17. Efficiency of the LCCL and LCL-T RI over the load range.

MOSFET $Q_{1}$ is highlighted in Fig. 15(b). The voltage and current divisions shown in the figure incorporate the scaling factor to denote actual values.

Fig. 16 demonstrate the fault tolerant operation of the LCCL RI. The step-change in the fault signal $\left(S_{f}\right)$ indicates the initiation of a low impedance fault in Fig. 16(a). The load current $i_{R}$ stabilizes itself after a few cycles of oscillation. Also, as the current gain $H_{i}$ of the LCCL RI increases with the reduction in $Q$ (Fig. 5(a)), the tank current $i_{s}$ reduces during the fault for a constant $i_{R}$. When the fault is cleared, as shown in Fig. 16(b), $i_{R}$ remains the same while $i_{s}$ increases to indicate an increase in the power drawn from the dc source. Thus, LCCL RI has inherent protection from short-circuit and overcurrent faults, which is highly beneficial for subsea operation that demands high reliability of the power converter.

\section{Efficiency Analysis}

Efficiency values of the LCCL and the LCL-T RI are plotted in Fig. 17 at two distinct operating frequencies, namely the

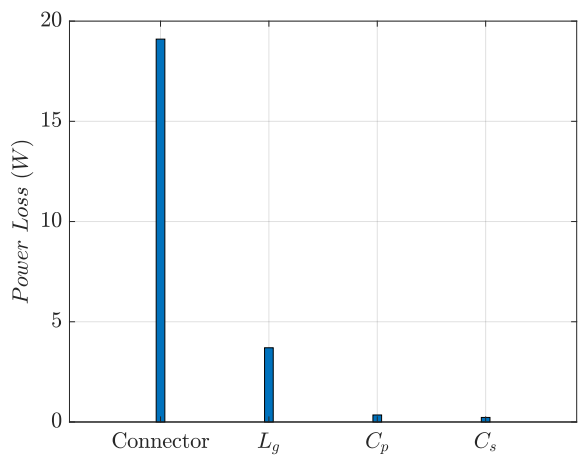

Fig. 18. Power loss distribution in LCCL RI at rated load $(250 \mathrm{~W})$.

resonant frequency $\left(f_{o}\right)$ and the peak current gain frequency $\left(f_{m}\right.$ or $\left.f_{r}\right)$ for a load resistance variation from $0.3 \Omega$ to 3.1 $\Omega$. Corresponding maximum output power is around $250 \mathrm{~W}$ to comply with the power rating of the load resistor assembly. The efficiency is computed as

$$
\eta=\frac{I_{R_{r}}^{2}\left(R+r_{L}\right)}{V_{g} I_{g}} \times 100 \%
$$

where $r_{L}$ represents the ESR of the load inductor. Please note that the hysteresis loss is not considered due to insufficient manufacturer data for the discrete inductors used in the experiment.

Both the LCCL and LCL-T RI exhibit low efficiency for the resonant frequency operation. This is due to a substantial reduction in current gain and increase in power factor angle, as observed in Fig. 12 and Fig. 13. The efficiency is notably enhanced for the peak current gain operation. Although the LCL-T RI manifests comparatively higher efficiency, the output power is relatively low. For a load resistance of $3.1 \Omega$ and dc input voltage of $50 \mathrm{~V}$, the power output of the LCL-T $\mathrm{RI}$ is only $95 \mathrm{~W}$, while that of the LCCL RI is $246 \mathrm{~W}$. To realize the same power output, the LCL-T RI requires much larger load resistance, leading to a lower efficiency because $H_{i}$ reduces and $\varphi_{i}$ increases with increasing $Q$, as per Fig. 7. Meanwhile, the LCCL RI manifests above $90 \%$ efficiency over a substantial load range at a higher operating frequency, which is suitable for high-frequency DEH application.

The power loss distribution in the LCCL RI, operating at the peak current gain, is evaluated at rated load and is depicted in Fig. 18. As the experimental assembly consists of discrete passive components, the power loss in the ESR of the connecting wires becomes the principal loss component. This stray loss could be minimized through a PCB implementation. The other major power loss component is the conduction power loss in the ESR of the tank inductor $L_{r}$, which depends on the magnitude of $I_{s_{r}}$. The turn-off switching loss is negligible owing to the relatively low switching frequency.

\section{CONCLUSions}

This paper presents an LCCL resonant inverter (RI) for high-frequency, constant current operation in direct electrical heating (DEH) systems. The best possible performance at nonimmittance for an LCCL RI is realized by tuning the tank 
components at the frequency of the peak current gain. The peak current gain based design minimizes the rms current and the total VA rating of the tank and enables ZVS of the inverter MOSFETs. Detailed analysis and design guidelines are presented for the LCCL RI. Experimental results on a laboratory prototype demonstrates the inverter performance and validates the design. In addition, an exhaustive comparison with the widely utilized LCL-T topology is provided to show the advantages of the LCCL RI in DEH or similar applications with high load inductance. The hardware-in-the-loop results are also presented for a high power LCCL RI to demonstrate the aforementioned advantages along with fault tolerant operation.

\section{REFERENCES}

[1] A. Ray and K. Rajashekara, "A resonant current regulator for direct electrical heating of subsea pipelines," in 2020 IEEE Energy Conversion Congress and Exposition (ECCE), pp. 5256-5262, 2020.

[2] A. Nysveen, H. Kulbotten, J. K. Lervik, A. H. Bornes, M. HoyerHansen, and J. J. Bremnes, "Direct electrical heating of subsea pipelinestechnology development and operating experience," IEEE Transactions on Industry Applications, vol. 43, no. 1, pp. 118-129, 2007.

[3] P. Angays, "High efficiency heating method for subsea pipelines heating," in 2011 Record of Conference Papers Industry Applications Society 58 th Annual IEEE Petroleum and Chemical Industry Conference (PCIC), pp. 1-8, 2011.

[4] C. Candelier, S. Durica, and F. Beys, "Subsea pipeline electrical heat trace (eht) - active heating - application for a deep water brown field development," in 2015 Offshore Mediterranean Conference, pp. 1-37, March 2015

[5] J. K. Larvik, A. H. Bornes, A. Nysveen, and M. Hoyer-Hansen, "Electromagnetic modelling of steel pipelines for deh applications," in International Society of Offshore and Polar Engineers, pp. 1-5, July 2007.

[6] A. Chen, A. Nysveen, M. HÃ syer-Hansen, and J. Lervik, "Analytical and fem calculation of electrical parameters of carbon steel pipe in deh systems," in 2018 IEEE International Magnetics Conference (INTER$M A G)$, pp. 1-5, April 2018.

[7] A. Chen, A. Nysveen, J. Lervik, and M. HÃ, yer-Hansen, "Prediction of heating power in magnetic pipe conducting large ac current with high frequencies up to 200hz.," in 2018 IEEE International Magnetics Conference (INTERMAG), pp. 1-1, April 2018.

[8] J. Song-Manguelle, M. H. Todorovic, R. K. Gupta, D. Zhang, S. Chi, L. J. GarcÃ@s, R. Datta, and R. Lai, "A modular stacked dc transmission and distribution system for long distance subsea applications," IEEE Transactions on Industry Applications, vol. 50, pp. 3512-3524, Sep. 2014.

[9] A. Ray, K. Rajashekara, and H. Krishnamoorthy, "Novel hvdc power transmission architectures for subsea grid," in 2019 Offshore Technology Conference, pp. 1-8, May 2019.

[10] W. Zhang and C. C. Mi, "Compensation topologies of high-power wireless power transfer systems," IEEE Transactions on Vehicular Technology, vol. 65, no. 6, pp. 4768-4778, 2016.

[11] Z. Pantic, S. Bai, and S. M. Lukic, "Zcs lcc-compensated resonant inverter for inductive-power-transfer application," IEEE Transactions on Industrial Electronics, vol. 58, no. 8, pp. 3500-3510, 2011.

[12] J. Feng, M. Fu, Q. Li, and F. C. Lee, "Resonant converter with coupling and load independent resonance for omnidirectional wireless power transfer application," in 2017 IEEE Energy Conversion Congress and Exposition (ECCE), pp. 2596-2601, Oct 2017.

[13] M. Khatua, A. Kumar, V. Yousefzadeh, A. Sepahvand, M. Doshi, D. Maksimovic, and K. K. Afridi, "High-performance megahertzfrequency resonant dc-dc converter for automotive led driver applications," IEEE Transactions on Power Electronics, vol. 35, no. 10, pp. 10396-10412, 2020.

[14] T. Saha, A. C. Bagchi, and R. A. Zane, "Analysis and design of an lcl-t resonant dc-dc converter for underwater power supply," IEEE Transactions on Power Electronics, vol. 36, no. 6, pp. 6725-6737, 2021.
[15] H. Wang, T. Saha, B. Riar, and R. Zane, "Design considerations for current-regulated series-resonant converters with a constant input current," IEEE Transactions on Power Electronics, vol. 34, no. 1, pp. 141-150, 2019.

[16] M. Borage, K. V. Nagesh, M. S. Bhatia, and S. Tiwari, "Resonant immittance converter topologies," IEEE Transactions on Industrial Electronics, vol. 58, no. 3, pp. 971-978, 2011.

[17] M. Borage, S. Tiwari, and S. Kotaiah, "Analysis and design of an lcl-t resonant converter as a constant-current power supply," IEEE Transactions on Industrial Electronics, vol. 52, pp. 1547-1554, Dec 2005.

[18] M. Borage, S. Tiwari, and S. Kotaiah, "Lcl-t resonant converter with clamp diodes: A novel constant-current power supply with inherent constant-voltage limit," IEEE Transactions on Industrial Electronics, vol. 54, no. 2, pp. 741-746, 2007.

[19] M. Borage, K. V. Nagesh, M. S. Bhatia, and S. Tiwari, "Design of lcl-t resonant converter including the effect of transformer winding capacitance," IEEE Transactions on Industrial Electronics, vol. 56, no. 5 pp. 1420-1427, 2009.

[20] H. Pollock, "Simple constant frequency constant current load-resonant power supply under variable load conditions," Electronics Letters, vol. 33, pp. 1505-1506, 1997.

[21] S. Dieckerhoff, M. Ruan, and R. De Doncker, "Design of an igbt-based lcl-resonant inverter for high-frequency induction heating," in Conference Record of the 1999 IEEE Industry Applications Conference. ThirtyForth IAS Annual Meeting (Cat. No.99CH36370), vol. 3, pp. 2039-2045 vol.3, 1999.

[22] J. M. Espi-Huerta, E. J. Dede Garcia Santamaria, R. Garcia Gil, and J. Castello-Moreno, "Design of the 1-lc resonant inverter for induction heating based on its equivalent sri," IEEE Transactions on Industrial Electronics, vol. 54, pp. 3178-3187, Dec 2007.

[23] S. Chudjuarjeen, A. Sangswang, and C. Koompai, "An improved 1lc resonant inverter for induction-heating applications with asymmetrical control," IEEE Transactions on Industrial Electronics, vol. 58, no. 7, pp. 2915-2925, 2011.

[24] R. W. Erickson and D. Maksimovic, Fundamentals of Power Electronics, 3rd Ed. New York: Springer, 2020.

[25] M. K. Kazimierczuk and D. Czarkowski, Resonant Power Converters, 2nd Ed. New York: Wiley, 2011. 